\title{
La música valenciana en la pimera mitad del siglo XX: necesidad de una nueva contextualización en la música española de su tiempo
}

\author{
Valencian Music in the First Half of the Twentieth Century: A Need for a \\ New Contextualization in Spanish Music of its Time
}

\author{
J. Pascual Hernández Farinós \\ Conservatorio Superior de Música de Valencia \\ jphfarinos@hotmail.com \\ ORCID iD: https:/ /orcid.org/0000-0002-2485-0355
}

\begin{abstract}
RESUMEN
La música española del siglo XX ha sido narrada siguiendo unas pautas historiográficas que, pese a su aceptación general, plantean dificultades para incluir toda la complejidad cultural y artística de este periodo, desvelada por recientes investigaciones musicológicas. Estas dificultades son también aplicables a la música valenciana de aquel siglo XX, por lo que este texto intenta plantear alternativas a esta narración, fruto de la experiencia del propio autor al manejar fuentes bibliográficas en la elaboración de su Tesis Doctoral.
\end{abstract}

Palabras clave: siglo XX, música española, música valenciana, historiografía.

\begin{abstract}
Spanish music of the twentieth century has been narrated following historiographical guidelines that, despite their general acceptance, propose difficulties to include all cultural and artistic complexity of this period revealed by recent musicological research. These difficulties are also applicable to Valencian music of the twentieth century, and therefore this article attempts to propose alternatives to this narration, as a result of the author's own experience in using bibliographical sources in his $\mathrm{Ph}$. D. Thesis.
\end{abstract}

Key words: Twentieth century, Spanish music, Valencian music, historiography. 


\section{LA MÚSICA VALENCIANA EN LA PIMERA MITAD DEL SIGLO XX: NECESIDAD DE UNA NUEVA CONTEXTUALIZACIÓN EN LA MÚSICA ESPAÑOLA DE SU TIEMPO}

Hernández Farinós, J. P. (2018). La música valenciana en la pimera mitad del siglo XX: necesidad de una nueva contextualización en la música española de su tiempo. Cuadernos de Investigación Musical, 2018, 6 (extraordinario), 352-359. doi: 10.18239/invesmusic.v0i0.1951

La necesidad de sistematizar la narración del devenir histórico es una tarea ardua y difícil, tanto en la selección de todo aquello que se pueda considerar relevante, como por la elección de un enfoque que sea apropiado para abarcar el conjunto de hechos a tratar. En el caso de la música española del pasado siglo XX, se han sucedido varios intentos que van desde el pionero La música contemporánea en España de Adolfo Salazar (Salazar, 1930), publicado en $1930^{1}$, hasta el más reciente del volumen 7 de la Historia de la Música Española, editado en 2013 por el Fondo de Cultura Económica (González Lapuente, 2013). En todos ellos se aprecian dos perspectivas generales a la hora de organizar todo el material historiable: clasificación por realidades geográficas en las que se aprecia el manejo de los conceptos fundamentales de nacionalismo y regionalismo como soporte para los criterios de organización de la realidad musical, tomando como base para articular su discurso la centralidad de Madrid y en menor medida Barcelona; clasificación por generaciones de compositores, en la que se intenta encasillar a cada autor en una época o subperiodo de tiempo, coincidiendo con su etapa de mayor fecundidad creativa o con la que mejor se lo encasilla en un discurso ya preconcebido (Franco, 1987: 35-37). Esta segunda perspectiva adolece de un exceso de rigidez, puesto que clasifica monolíticamente a cada compositor en un breve lapsus de tiempo, lo que dificulta una visión global de su obra y deja al margen cualquier evolución estilística que hubiera podido experimentar a lo largo de su vida.

Este esquema parece claro y fácilmente comprensible, en tanto que articula un discurso aparentemente cerrado y sin puntos débiles en su narración. De hecho, las principales obras de Federico Sopeña (Sopeña, 1976), Manuel Valls Gorina (Valls Gorina, 1960) (Valls Gorina, 1962), Antonio Fernández-Cid (Fernández Cid, 1949) (Fernández Cid, 1963) (Fernández Cid, 1973) y Tomás Marco (Marco: 1970) (Marco: 1983), plasman este discurso creando un edificio conceptual de fácil asimilación, muy útil para todo aquel interesado en una visión panorámica de la creación musical de este siglo.

Sin embargo, desde los pasados años ochenta han surgido una serie de estudios más detallados y pormenorizados en torno a este periodo histórico, tanto acerca de compositores en particular como de contexto musical en general, que generaron en un principio dudas respecto a la validez de este esquema. Con el paso del tiempo, estas dudas se han incrementado e incluso en algunas obras como Música española entre dos guerras, 19141945 (Suárez-Pajares, 2002: 9-17), se reconocen las dificultades y se cuestiona este esquema historiográfico.

\footnotetext{
${ }^{1}$ Reeditado en 1982 por la Universidad de Oviedo.
} 


\section{J. PASQual HeRnàndez Farinós}

En el caso valenciano, las primeras iniciativas en crear una historia de la música valenciana son muy tardías, datadas a finales de los años setenta, de la mano de José Climent (Climent, 1978) y Eduardo López-Chavarri Andújar (Doménech y López-Chavarri Andújar, 1978). En aquel momento, se advertía la carencia de estudios detallados que enriquecieran tanto la información como la articulación de este discurso, los cuales no empezaron a plasmarse hasta finales de la década de los ochenta, como es el caso de obras posteriores de los arriba citados José Climent (Climent, 1989) y Eduardo López-Chavarri Andújar (López-Chavarri Andújar, 1985) (López-Chavarri Andújar, 1992). A ellos cabe añadir aportaciones de signo enciclopédico como las de Bernardo Adam Ferrero (Adam Ferrero, 1988), así como las de José Luis García del Busto (García del Busto, 1992) y Vicente Galbis López (Galbis, 1992), en las que se advierte un enfoque más completo e interdisciplinar.

Esta situación generó ciertos problemas en épocas como el aquí tratado siglo XX, puesto que los citados autores intentaron incardinar la música valenciana en el discurso general arriba citado, aplicado a la música española del mencionado siglo. En estas circunstancias, se trató esta música desde una perspectiva local frente a los grandes núcleos culturales de la época como eran Madrid y Barcelona, dando como resultado una visión aparentemente ordenada pero establecida a priori, de cuestionable validez en tanto que podría ser rebatida por futuros estudios detallados sobre autores y realidades musicales, entonces todavía en proyecto.

Este problema se amplió todavía más en el caso de la clasificación de autores según una perspectiva generacional, puesto que en la música valenciana del siglo XX abundan los casos de compositores particularmente longevos como Eduardo López-Chavarri Marco u Óscar Esplá. Incluso en compositores de menor longevidad que los citados, como Joaquín Rodrigo o Manuel Palau, también se aprecia la fragilidad de este esquema, puesto que ambos son tratados como compositores de los años de la Posguerra, puesto que fue en esta época cuando sus figuras gozaron de mayor relevancia artística y social. Sin embargo, al ignorar la totalidad de su trayectoria creativa se ofrece una visión muy sesgada de sus figuras y obras, como es el caso de su producción en los años veinte y treinta, cuando la influencia de sus estudios en Paris dio paso a unas obras de corte casi vanguardista, cuya incidencia se reflejó abiertamente en el contexto musical valenciano. Comparando ambas épocas, un análisis detallado de sus respectivas obras muestran dos realidades artísticas distintas, pero fruto de dos épocas diferentes, aunque cabe preguntarse porqué se incluye una en la narrativa histórica española de la época y se deja de lado la otra, puesto que con ésta la visión de la obra y estilo de ambos podría cambiar considerablemente.

Como ejemplo de este proceso de reflexión, se va a poner el ejemplo de las obras de Joaquín Rodrigo en la Valencia de los años veinte que han sido clasificadas como neoclásicas. En este contexto cabe citar como primera obra destacada su Suite para piano (1923) estrenada en Paris por Ricardo Viñes en 1928 y sus Juglares (1923) para orquesta de notoria modernidad, que acabó cristalizando en sus Cinco Piezas Infantiles (1924) para orquesta que recibieron una Mención Honorífica en el Premio Nacional de Música de 1925. Sin embargo, estas obras quedan eclipsadas por la visión historiográfica tradicional que empieza a apreciar su obra con la Zarabanda lejana y Villancico (1925-1930), como se 


\section{LA MÚSICA VALENCIANA EN LA PIMERA MITAD DEL SIGLO XX: NECESIDAD DE UNA NUEVA CONTEXTUALIZACIÓN EN LA MÚSICA ESPAÑOLA DE SU TIEMPO}

observa en los textos de Federico Sopeña (Diego, Rodrigo y Sopeña, 1949), frente a la más positiva valoración de la más reciente obra de Antonio Gallego (Gallego, 2003: 21-59). Por otra parte, a la hora de encasillar su producción posterior como Neo-casticista, se toma como base sus Cuatro Piezas para piano-Cuatro Danzas de España (1937), cuya primera pieza titulada Calesera en homenaje a Federico Chueca dio título a esta etiqueta estilística, pero obviando que la cuarta pieza titulada Danza Valenciana emplea la melodía popular valenciana titulada "L’ú i el dos" (Gallego, 2003: 110-114). En conjunto, se aprecia un proceso de selección de obras y momentos creativos adecuados a un esquema preconcebido, sin valorar la totalidad de su producto, incluso en momentos coincidentes.

Si tomamos el caso de Manuel Palau, se deja en segundo plano toda su producción de los años veinte y treinta, cuando en la misma se aprecia un carácter más fresco e innovador, pero lejano al esquema historiográfico que se intentó crear en torno a su persona. De este modo, se observa la presencia de obras politonales como las Tres Impresiones Orquestales $(1924)^{2}$, así como obras destacables en las que realiza una interesante mixtura de música popular valenciana con lenguajes impresionistas parisinos como se aprecia en Poemas de Juventud (1925), Siluetas (1927), Cuarteto en estilo popular (1928) y Poemes de Llum (1935); sólo se cita su Gongoriana (1927) en razón de que fue galardonada con el Premio Nacional de Música de 1927, pero sin incidir en su influencia estilística. Por otra parte, su deriva hacia un Neo-Clasicismo innovador apreciable en su Divertimento (1937) y la Mascarada Sarcástica (1939) queda totalmente eclipsado ante su producción de Posguerra, de corte más conservador. Aunque la figura de Manuel Palau se engrandeció durante aquellos años, sobre todo a nivel administrativo e institucional, no resulta justificable que se priorice su producción de los años cuarenta y cincuenta por encima de la realizada en periodos precedentes, incluso a pesar de que su figura ha sido objeto de Tesis Doctorales como la de Salvador Seguí, además de publicaciones del mismo autor (Seguí, 1994) (Seguí, 1999).

En cambio, los compositores alicantinos Rafael Rodríguez Albert y Óscar Esplá están mejor caracterizados por la historiografía tradicional, en gran parte porque lo más notorio de su producción fue compuesto y estrenado en el entorno musical y cultural de Madrid, altavoz muy adecuado para su obra, pese a que siempre se calificaron a sí mismos como 'compositores mediterráneos'.

En estas circunstancias, parece mucho más operativo plantear una historia de la música española del siglo XX por periodos, siguiendo la evolución de los estilos musicales predominantes en cada uno de ellos, insertando como ejemplos obras de autores concretos en cada momento, más que compartimentarlos forzadamente en un discurso generacional. Bajo esta pauta, la obra y el estilo de Joaquín Rodrigo y Manuel Palau serían tratados de una forma más precisa y adecuada a su realidad creativa en el contexto valenciano y español. Por otra parte, la pertenencia a escuelas regionales y nacionales debe ser tratada

\footnotetext{
2 Para una valoración de la importancia de esta obra ver: Hernández Farinós, J. P. "Tres Impresiones Orquestales de Manuel Palau: Lenguajes vanguardistas en la Valencia de los años 20". Musicología en el siglo XXI: nuevos retos, nuevos enfoques. Madrid: Sedem (en prensa).
} 


\section{J. PASQUal HeRnàndez Farinós}

con mucha cautela, en tanto que el siglo XX fue una época en la que se alternó con cierta volubilidad el sentimiento nacionalista con unos tiempos en que los medios de comunicación permitieron una apertura de miras que hicieron tanto o más factible una perspectiva cosmopolita y universal de la cultura y la música.

Respecto a la historia de la música valenciana, el último intento de articular un discurso de este tipo tuvo lugar en el año 1992, cuando la Editorial Prensa Valenciana publicó en fascículos acompañados de grabaciones una producción de este tipo, coordinada por Gonzalo Badenes (Badenes, 1992). Sin embargo, en aquel entonces todavía no se tenía la posibilidad de articular un discurso diferente del arriba establecido, tanto por el desigual conocimiento de las diferentes épocas tratadas como por la gran dificultad en coordinar especialistas surgidos de diferentes escuelas historiográficas y musicológicas. Por otra parte, ya han pasado más de veinticinco años desde su publicación, tiempo en el que las investigaciones y estudios sobre la música valenciana se han incrementado exponencialmente, mientras que los enfoques se han enriquecido considerablemente, dando la opción de ofrecer un discurso más rico y cercano a la auténtica realidad histórica de la música valenciana. Por esta razón, creo que es ya necesaria la realización de una nueva historia de la música valenciana, en la que se aporte toda la información extraída en las numerosas investigaciones realizadas en estos últimos años, dando lugar a una obra que podría ser bastante diferente a aquella última iniciativa.

En mi caso particular, realicé una Tesis Doctoral titulada La composición orquestal valenciana a través de la aportación del Grupo de los Jóvenes (1925-1960), dirigida por Vicente Galbis y José Martín, leída en la Universitat de València en enero de 2011 (Hernández, 2011), tras un largo proceso de investigación que se prolongó durante once años. En la misma obtuve una importante cantidad de información sobre la música valenciana de aquellos años, cuya realidad era tratada de forma parcial e incompleta en las obras de Climent, López-Chavarri Andújar y en la Historia de la Música Valenciana de la Ed. Prensa Valenciana. Todo ello repercutió en su desconocimiento tanto en las diferentes historias de la música española, como incluso en la más reciente publicada por el Fondo de Cultura Económica en 2013.

En la misma tuve la opción de confrontar el resultado de mis investigaciones con las diferentes perspectivas historiográficas utilizadas durante el pasado siglo, encontrándome con un casi completo desconocimiento de su trayectoria como Grupo, y una visión parcial y sesgada de sus figuras individuales. Sólo en la Historia de la Música española e hispanoamericana, publicada por José Subirá en 1953, se cita al Grupo (Subirá, 1953: 819820), quizás porque el autor ejerció durante los años treinta como crítico en la revista Musicografía ${ }^{3}$ donde se trató con frecuencia la obra de los miembros del Grupo.

El Grupo de los Jóvenes estaba integrado por Vicente Asencio, Vicent Garcés, Ricardo Olmos, Luis Sánchez y Emilio Valdés; autores de posterior trayectoria muy variopinta, pero con un punto común en la recepción del magisterio de Manuel Palau en la

\footnotetext{
${ }^{3}$ Revista de carácter musical publicada en la localidad de Monóvar (Alicante), con periodicidad mensual entre 1933 y 1936. En la misma publicaron reseñas y críticas personajes de la talla de José Subirá.
} 


\section{LA MÚSICA VALENCIANA EN LA PIMERA MITAD DEL SIGLO XX: NECESIDAD DE UNA NUEVA CONTEXTUALIZACIÓN EN LA MÚSICA ESPAÑOLA DE SU TIEMPO}

Valencia de finales de los años veinte y principios de los treinta (a excepción de Vicente Asencio), paso previo a la publicación de su Manifiesto en enero de 1934, con el que dieron muestra de una base estética de signo nacionalista valenciano y su intención de incidir en la vida musical valenciana de la época.

Para poder confrontar esta base de investigación con el esquema historiográfico español antes citado, voy a exponer la forma en que Tomás Marco los citó en su Historia de la Música Española-Siglo XX, publicada por Alianza Editorial en 1983. En la misma, se cita a Ricardo Olmos como integrante de la Generación del 27, dentro del sub-apartado de 'otras figuras y asimilados' (Marco, 1983: 147), probablemente por su fecha de nacimiento en 1905 que hizo que se lo encajara forzadamente en ese periodo de la historia de la cultura, cuando en aquellos años todavía estaba completando su formación compositiva con Palau. En el caso de Vicent Garcés, se lo califica como 'músico posterior a la Guerra Civil y continuista' (Marco, 1983: 188), probablemente influido por la visión historiográfica que había ofrecido veinte años antes Manuel Valls Gorina (Valls Gorina, 1960: 22-23) (Valls Gorina, 1962: 192, 210-211, 226). Vicente Asencio queda encasillado dentro de la etiqueta de 'Nacionalismo Casticista' (Marco, 1983: 182-183), probablemente por su obra posterior a los años cuarenta donde está presente la huella de Manuel de Falla, pero no como un rasgo exclusivo con el que se pueda definir con precisión su persona y obra. Por otra parte, la figura de Asencio fue encuadrada por Valls Gorina dentro de una llamada Generación de la República, sin apenas tener constancia de la obra que compuso durante aquellos años (Valls Gorina, 1960: 111) (Valls Gorina, 1962: 93). Finalmente, las figuras de Luis Sánchez y Emilio Valdés quedan totalmente al margen y sin citar, probablemente porque su desaparición de los grandes centros musicales de la época tras la Guerra Civil hizo imposible dar a conocer su música, pero recientes iniciativas de recuperación de su obra han desvelado la calidad de sus respectivas producciones. En definitiva, queda claro y demostrado que el esquema historiográfico tradicional no ofrecía una respuesta a la realidad musical, en gran parte porque estos historiadores desconocían una gran cantidad de información que ha surgido en investigaciones recientes, lo que justifica la necesidad de una nueva articulación de la narración histórica.

Soy de la opinión de que el mismo proceso de ampliación y enriquecimiento se podría aplicar al resto de periodos históricos, por lo que una nueva Historia de la Música Valenciana lo actualizaría y generaría una base conceptual perfectamente utilizable en las futuras Historias de la Música Española. 


\section{J. PASQUal HeRnàndez Farinós}

\section{BIBLIOGRAFÍA}

Adam Ferrero, B. (1988). Músicos Valencianos. 2 vol. Valencia: Promotora Internacional de Publicaciones (PROIP).

Badenes, G. (ed.) (1992). Historia de la música de la Comunidad Valenciana. Valencia: Editorial Prensa Alicantina-Prensa Valenciana.

Climent, J. (1978). Historia de la música contemporánea valenciana. Valencia: Del Cenia al Segura.

Climent, J. (1989). Historia de la Música Valenciana. Valencia: Rivera Mota.

Diego, G. Rodrigo, J. y Sopeña, F. (1949). Diez años de música en España. Musicología, Intérpretes. Compositores. Madrid: Espasa-Calpe.

Doménech, J. y López-Chavarri Andújar, E. (1978) 100 años de Música Valenciana (18781978). Valencia: Caja de Ahorros de Valencia.

Fernández Cid, A. (1949). Panorama de la Música en España. Madrid: Editorial Dossat.

Fernández Cid, A. (1963). La música y los músicos españoles en el siglo XX. Madrid: Ed. Cultura Hispánica.

Fernández Cid, A. (1973). La música española en el siglo XX. Madrid: Fundación Juan March.

Franco, E. (1987). “Generaciones musicales españolas”, en E. Casares (ed.) La música en la Generación del 27. Homenaje a Lorca, Madrid: Ministerio de Cultura.

Galbis López, V. (1992). "Una aproximació a la història de la música valenciana”. En VVAA, La Imatge de la Música en Sant Pius V, pp. 33-64. Valencia: Generalitat Valenciana.

Gallego, A. (2003). El arte de Joaquin Rodrigo. Madrid: Iberautor Promociones Culturales.

García del Busto, J. L. (1992). "La música del siglo XX anterior a la guerra civil”. En Badenes, G. (ed.). Historia de la Música de la Comunidad Valenciana, pp. 361-380. Valencia: Editorial Prensa Alicantina-Prensa Valenciana.

González Lapuente, A. (ed.) (2013), Historia de la Música en España e Hispanoamérica (Vol. 7): La Música en España en el siglo XX. Ed. Alberto González Lapuente. Madrid: Fondo de Cultura Económica. 


\section{LA MÚSICA VALENCIANA EN LA PIMERA MITAD DEL SIGLO XX: NECESIDAD DE UNA NUEVA CONTEXTUALIZACIÓN EN LA MÚSICA ESPAÑOLA DE SU TIEMPO}

Hernández Farinós, J. P. (2011). La composición orquestal valenciana, a través de la aportación del Grupo de los Jóvenes (1925-1960) (Tesis Doctoral). Valencia: Universitat de ValènciaE.G.

López-Chavarri Andújar, E. (1985). Breviario de Historia de la Música Valenciana. Valencia: Piles.

López-Chavarri Andújar, E. (1992). Compositores valencianos del siglo XX. Valencia: Generalitat Valenciana-Música 92.

Marco, T. (1970). La música de la España Contemporánea. Madrid: Publicaciones españolas.

Marco, T. (1983). Historia de la música española, vol. 6: Siglo XX. Madrid: Alianza Editorial.

Salazar, A. (1930). La música contemporánea en España. Madrid: Ediciones La Nave. Reedición en 1982, Oviedo: Universidad de Oviedo.

Seguí, S. (1994). La praxis armónico-contrapuntística en la obra liedística de Manuel Palau. Vida y obra del músico valenciano (Tesis Doctoral). Valencia: Universitat de València-E.G.

Seguí, S. (1999). Manuel Palau (1893-1967). Valencia: Consell Valencià de Cultura.

Sopeña, F. (1976). Historia de la música española contemporánea. Madrid: Rialp.

Suárez-Pajares, J. (ed.) (2002). Música española entre dos guerras, 1914-1945. Granada: Archivo Manuel de Falla.

Subirá, J. (1953). Historia de la música española e hispanoamericana. Barcelona: Salvat.

Valls Gorina, M. (1960). La Música Catalana Contemporània. Barcelona: Ed. Selecta.

Valls Gorina, M. (1962). La música española después de Manuel de Falla. Madrid: Revista de Occidente. 